\title{
Optimum Device and Modulation Scheme Selection for Optical Wireless Communications
}

\author{
Hyunchae Chun, Member IEEE, Sujan Rajbhandari, Member IEEE, Grahame Faulkner, Enyuan Xie, Jonathan J. \\ D. McKendry, Erdan Gu, Member IEEE, Martin D. Dawson, Fellow IEEE, and Dominic O'Brien, Member IEEE
}

\begin{abstract}
There has been extensive modelling of the optical wireless channel, and the optimum modulation scheme for a particular channel is well-understood. However, this modelling has not taken into account the trade-offs that transmitter and receiver selection usually involve. For a particular type of transmitter, the modulation bandwidth and available power are closely related, as are receiver bandwidth, active area and sensitivity. In this paper, we present a design approach that takes this device selection into account. The paper details a general design method for an optical wireless communication system using a holistic design approach (i.e. considering channel, modulation schemes and device constraints). The paper shows results for particular examples, showing a substantial increase in margin (or data-rate) is available using this approach. For instance, by using this approach mutually optimising both schemes and device constraints, it is found that for an optimally chosen Gallium Nitride micro-LED and a commercial photo receiver pair, a $20 \mathrm{~dB}$ SNR margin (or $\sim 3$ times datarate improvement) can be obtained compared with a more typical approach concerning mainly on the scheme optimisation.
\end{abstract}

Index Terms - Optical Communications, VLC, Visible light communication, OWC, Optical wireless communication, OFDM, WLED, RC LED, Equalisation, Technology-curve, LiFi, micro-LED

\section{INTRODUCTION}

Optical wireless communication (OWC) and visible light communications (VLC) are rapidly growing research areas [1-4]. Recent research has focused on increasing the available data rate. These include work on high-level modulation, spatial-multiplexing, wavelength-multiplexing, and/or channel equalisation [5-6]. In the past several years, projects have investigated custom devices for VLC, such as in reference [7] where customised micro light-emitting diodes ( $\mu$ LEDs) with an order s of magnitude higher bandwidth than commercial LEDs and white LEDs (WLED) were investigated. There has been extensive work on different modulation schemes, comparing the bandwidth and power required for each. The method shown in [8] estimates the power and bandwidth requirements for different intensity modulation direct detection (IM-DD) schemes. However, it does not include the device and technology constraints at the transmitter and receiver, and does not consider

H. Chun is with Department of Information and Telecommunication Engineering, Incheon National University, Incheon, Korea. E-mail: Hyunchae.chun@inu.ac.kr

G. E. Faulkner, and D. C. O'Brien are with the Department of Engineering Science, University of Oxford, Oxford, UK. E-mail: \{grahame.faulkner, dominic.obrien\}@eng.ox.ac.uk.

S. Rajbhandari is with Huawei Technologies Sweden AB, Gothenburg, Sweden. E-mail: sujan@ieee.org. techniques compensating a band-limited channel, such as equalisation. To the best of our knowledge, we for the first time present a holistic design method optimising such parameters in the selection of the optimum communication scheme.

In section II, we introduce the 'Technology-curve' showing the available bandwidth and signal-to-noise ratio (SNR) tradeoff for a particular set of transmitter and receiver technologies. In section III, a detailed method to select the optimum pair of communication scheme and device parameters is presented. Two examples are shown to illustrate the method: A typical VLC lighting scenario, where illumination is combined with communication, as this has wide application, and a point-topoint communications link, of a type widely used to assess communications performance. This latter link example shows how the method can be used where there is limited choice in devices. Conclusions are drawn in section IV.

\section{TECHNOLOGY-CURVE}

\section{A. Motivation}

The available SNR for a particular bandwidth (BW) channel is a function of the transmitted power, link loss, and the receiver sensitivity. For a particular emitter technology, such as Gallium Nitride $(\mathrm{GaN})$ LEDs, there is often a trade-off between emitted power and modulation bandwidth. For instance, increasing the total active area of a standard LED can increase the maximum output power but reduce the modulation bandwidth by increasing the total capacitance. Another example is GaN $\mu L E D s$, which are LEDs with pixel dimensions $\leq 100 \mu \mathrm{m}$, where the pixel dimensions are typically small enough that capacitance effects do not dominate; instead the modulation bandwidth is determined by the injected carrier lifetime. Because smaller $\mu$ LEDs can be driven with higher current densities, due to reduced current crowding and improved heat dissipation [9], they can reach higher modulation bandwidths than their larger area equivalents as the carrier lifetime decreases with current density [7]. As a result the general trend for $\mu$ LEDs is also that smaller devices emit lower power but have larger bandwidths. Similarly, for a receiver such as a typical Silicon photodetector the bandwidth is inversely proportional to the detection area, as the device's capacitance and thus $R C$ constant increases with area.

E. Xie, J. J. D. McKendry, E. Gu, and M. D. Dawson are with the Institute of Photonics, Department of Physics, University of Strathclyde, Glassgow, U.K. E-mail: \{enyuan.xie, jonathan.mckendry, erdan.gu, m.dawson\}@strath.ac.uk.

We gratefully acknowledge support by the UK Engineering and Physical Sciences Research Council (EPSRC) under grant EP/K00042X/1.

The data is available online at http://dx.doi.org/10.21227/mkbr-tb67. 
(a) Source power vs bandwidth trade-off

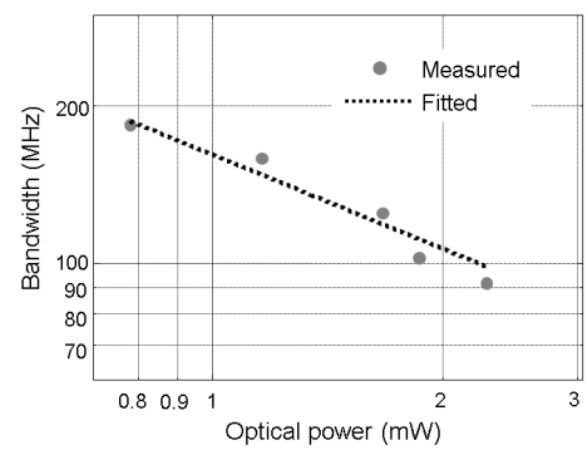

(b) Detector size vs bandwidth trade-off

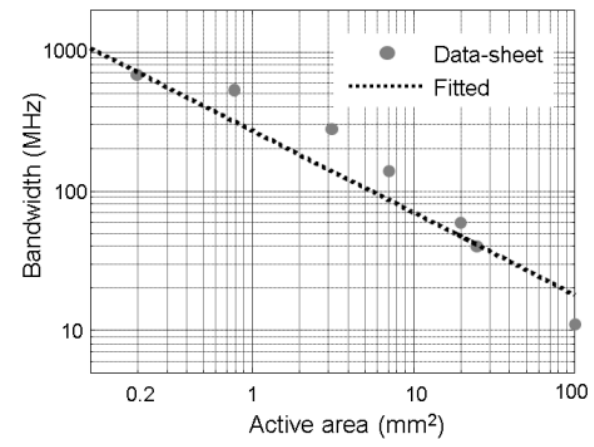

Fig. 1. (a) Source power-bandwidth trade-off from GaN $\mu$ LEDs with measured points and fitted line, and (b) detection area-bandwidth trade-off from Hamamatsu S8664 series with data points from datasheet and fitted line

However, increasing the detection area increases the power collected, equivalent to an increase in available SNR. There are a number of approaches used to maintain bandwidth whilst increasing detection area, most using arrays of receivers that are combined in different ways [10-12]. There is also work on waveguide receiver arrays (for instance [13]). Whilst the approach set out here assumes a typical relationship for a capacitance limited system, any parameterised relationship relating collection area and sensitivity to bandwidth can be used. So, for a particular set of technologies such as a GaN LED and Silicon photodetector the available SNR will depend on the bandwidth of the channel. If this technology dependent trade-off between BW and SNR (known in this paper as a Technology-curve) can be formulated, it becomes possible to optimise a communication system using a particular technology, achieving the highest SNR margin or the highest data-rate for a given channel bandwidth.

\section{B. Derivation of the Technology-curve}

To investigate the characteristic of light sources, $\mu$ LEDs are considered since they typically demonstrate a strong variation in modulation BW versus LED size, and are therefore suitable test cases to study [7]. Fig. 1 (a) shows the available source power $\left(P_{\mathrm{s}}\right)$ vs. the maximum $-3 \mathrm{~dB}$ (electrical) BW for a series of GaN $\mu$ LEDs. A power-law fit is used to generalise this result, so that

$$
P_{\mathrm{s}}=C_{\text {source }}\left(B W_{\text {source }}\right)^{-k_{\mathrm{s}}}
$$

Values of $\mathrm{C}_{\text {source }}=4.8 \times 10^{10}$ and $\mathrm{k}_{\mathrm{s}}=1.67$ brings the minimum squared error and is in reasonably good agreement with the measured results. Although the power-law fit is not the most accurate, it captures the power-bandwidth dependency well. More complex fitting over more limited ranges are options to increase fitting accuracy if required. The devices whose data is shown in Fig. 1(a) are from the same die and the same epitaxial wafer. However, the size is different, and it means different RC time constant and current density, both affecting the BW. Fig. 1 (b) shows the detection area and BW trade-off for a particular set of commercial detectors (Hamamatsu S8664 series). A similar power-law fit is used to relate bandwidth to the detection area.

$$
A_{\mathrm{d}}=C_{\text {detector }}\left(B W_{\text {detector }}\right)^{-\mathrm{k}_{\mathrm{d}}}
$$

As shown in the Fig. 1 (b), $C_{\text {detector }}=2 \times 10^{8}$ and $k_{\mathrm{d}}=1.69$ represent the relationship reasonably well. Then, the effect of these trade-offs on actual SNR and BW is investigated.
The received electrical signal amplitude $\left(\mathrm{S}_{\text {received }}\right)$ for a typical optical link is given by (3).

$\mathrm{S}_{\text {received }}=$

$$
\overbrace{\frac{m+1}{2 \pi} P_{s} \cos ^{\mathrm{m}}(\theta)}^{\begin{array}{c}
\text { Lambertian } \\
\text { intensity }
\end{array}} \cdot \overbrace{\cos (\Phi) \frac{G_{\mathrm{opt}, \mathrm{rx}} A_{d}}{D^{2}}}^{\begin{array}{c}
\text { Effective } \\
\text { solid angle }
\end{array}} \overbrace{M \cdot R \cdot G_{\mathrm{amp}}}^{\begin{array}{c}
\text { Receiver's } \\
\text { gain }
\end{array}}
$$

Equation (3) consists of three terms: Lambertian intensity from the source, the receiver's effective solid angle and its gain (from optical power to voltage). For the radiant Lambertian intensity, $\theta$ is the radiating angle and $m$ is the Lambertian order [6], with the total radiant flux $\left(P_{\mathrm{s}}\right)$. For a receiver with a photo sensitive area $\left(A_{\mathrm{d}}\right)$, located at distance $\mathrm{D}$ away from the source, the effective solid angle is determined by the receiving angle $(\Phi)$ and the receiver optics gain $\left(G_{o p t, r x}\right)$. The received optical power is then converted to an electrical signal by receiver's responsivity $(R)$, typically followed by a transimpedance structure with a gain of $G_{a m p} . M$ represents an avalanche gain when avalanche photo diodes (APD) are considered (otherwise $M=1$ ). The electrical SNR can be estimated as (4.1) and (4.2). The first noise term $\left(N_{\text {dep }}\right)$ consists of a signal dependent shot-noise term, with an allowance for excess noise if the receiver uses an APD. The second noise term $\left(N_{\text {ind }}\right)$ includes all other signal independent noise such as the thermal noise and additional noise from the receiver chain.

$$
\begin{aligned}
\mathrm{SNR} & =\frac{S_{\text {received }}^{2}}{2 q S_{\text {received }} M^{2+x} B W+N_{\text {ind }} \mathrm{BW}} \\
& =\frac{C_{\text {link }}^{2}\left(P_{s} \cdot A_{d}\right)^{2} G_{\mathrm{r}}^{2}}{N_{\mathrm{dep}}\left(P_{s} \cdot A_{d}\right) B W+N_{\text {ind }} B W}
\end{aligned}
$$

, where

$$
C_{\text {link }}=\frac{m+1}{2 \pi} \cos ^{\mathrm{m}}(\theta) \cos (\Phi) \frac{G_{\mathrm{opt}, \mathrm{rx}}}{D^{2}}
$$

, $G_{\mathrm{r}}=M \cdot R \cdot G_{\mathrm{amp}}$, and $\mathrm{q}$ is the electronic charge, and $\mathrm{x}$ is the excess noise index. In this case $B W$ represents the receiver bandwidth, and in the subsequent analysis, it is assumed that $B W_{\text {detector }}=\left(B W_{\text {source }}\right)=B W$. For a particular technology where there is design freedom optimum performance will be achieved when the bandwidths are matched so this is the case chosen here. With restricted device availability worst-case modelling is still feasible by setting the total $\mathrm{BW}=\min$ 


$$
\begin{aligned}
& \mathrm{SNR}_{\text {gain }}=\frac{\mathrm{SNR}_{1}}{\mathrm{SNR}_{0}}=\frac{\frac{\mathrm{C}_{\text {link }}^{2}\left(\mathrm{P}_{s, 0}\left(\frac{\mathrm{BW}_{1}}{B W_{0}}\right)^{-k_{s}} \cdot A_{d, 0}\left(\frac{\mathrm{BW}_{1}}{B W_{0}}\right)^{-k_{d}}\right)^{2} \mathrm{G}_{\mathrm{r}}^{2}}{\mathrm{~N}_{d e p}\left(\mathrm{P}_{s, 0}\left(\frac{\mathrm{BW}_{1}}{B W_{0}}\right)^{-k_{s}} \cdot A_{d, 0}\left(\frac{\mathrm{BW}_{1}}{B W_{0}}\right)^{-k_{d}}\right) \mathrm{BW}_{1}+\mathrm{N}_{\text {ind }} \mathrm{BW}_{1}}}{\frac{\mathrm{C}_{\text {link }}^{2}\left(\mathrm{P}_{s, 0} \cdot A_{d, 0}\right)^{2} \mathrm{G}_{\mathrm{r}}^{2}}{\mathrm{~N}_{d e p}\left(\mathrm{P}_{s, 0} \cdot A_{d, 0}\right) \mathrm{BW}_{0}+\mathrm{N}_{\text {ind }} \mathrm{BW}_{0}}} \\
& =\left\{\begin{array}{l}
\left(\frac{\mathrm{BW}_{1}}{B W_{0}}\right)^{-k_{s}} \cdot\left(\frac{\mathrm{BW}_{1}}{\mathrm{~B} W_{0}}\right)^{-k_{d}} \cdot\left(\frac{\mathrm{BW}_{1}}{B W_{0}}\right)^{-1}=\Delta \mathrm{BW}^{-\left(K_{\text {system }}+1\right)}, \text { for signal dependent noise limit } \\
\left(\frac{\mathrm{BW}_{1}}{B W_{0}}\right)^{-2 k_{s}} \cdot\left(\frac{\mathrm{BW}_{1}}{B W_{0}}\right)^{-2 k_{d}} \cdot\left(\frac{\mathrm{BW}_{1}}{B W_{0}}\right)^{-1}=\Delta \mathrm{BW}^{-\left(2 K_{\text {system }}+1\right)}, \text { for signal independent noise limit }
\end{array}\right. \\
& \overline{\mathrm{SNR}}_{\text {gain }}= \begin{cases}\Delta \mathrm{BW}^{-K_{\text {system }}} & , \text { for signal dependent noise limit } \\
\Delta \mathrm{BW}^{-2 K_{\text {system }}} & , \text { for signal independent noise limit }\end{cases} \\
& \text {,where } \Delta \mathrm{BW}=\frac{\mathrm{BW}_{1}}{B W_{0}} K_{\text {system }}=k_{s}+k_{d}
\end{aligned}
$$

$\left(\mathrm{BW}_{\text {source }}, \mathrm{BW}_{\text {detector }}\right)$ since an additional $\mathrm{SNR}$ margin is expected from the non-limiting side. Equation (4.2) shows a typical SNR equation $[8,14]$ rewritten in terms of $P_{s}$ and $A_{d}$, which are the main controlling variables for the trade-off analysis. Equations (1) and (2) show the variation in $P_{S}$ and detector area $A_{d}$ with bandwidth. These can be combined with (4.2) to create a relationship that shows how SNR changes with BW.

Equation 5.1 shows how SNR gain between two different $\mathrm{BW}$ channels $\left(\mathrm{BW}_{0}\right.$ and $\left.\mathrm{BW}_{1}\right)$ created using the same technology $\left(\mathrm{SNR}_{\text {gain }}=\frac{\mathrm{SNR}_{1}}{\mathrm{SNR}_{0}}\right)$ is related to the change in $\mathrm{BW}$ of the channel $\left(\triangle \mathrm{BW}=\frac{\mathrm{BW}_{1}}{B W_{0}}\right)$. As shown in equation (5.2), the tradeoff can be simplified by assuming either only the signal dependent noise is significant, or that signal independent noise dominates. For both these cases the exponent term $\left(K_{\text {system }}=k_{s}+\right.$ $k_{d}$ ) governs the overall trade-off between $\mathrm{SNR}_{\text {gain }}$ and $\Delta \mathrm{BW}$. Therefore, when the $K_{\text {system }}$ from a particular set of devices is known, one can draw a trade-off curve representing a selection of different device sets.

Equation 5.2 has a $(\Delta \mathrm{BW})^{-1}$ power term in addition to $K_{\text {system. }}$. This is due to the reduction in effective noise $\mathrm{BW}$ as the $\mathrm{BW}$ changes. The noise $\mathrm{BW}$ is the $\mathrm{BW}$ over which the AWGN noise spectral density must be integrated to create noise power equal to the noise power of the system. The SNR-BW requirements curves, as derived in [8] for example, generally take this into account as they normalise to the same data-rate. In other words, the requirement curves effectively include the expected increase (or decrease) in SNR due to the BW decrease (or increase) by applying various multi-level modulation schemes. For this reason, equation 5.3 defines $\overline{\mathrm{SNR}}_{\text {gain }}$ with the $\Delta \mathrm{BW}^{-1}$ term removed, so that only additional gain in SNR due to the transmitter and receiver technology changes with BW reduction is taken into account. We define the line drawn by the $\overline{\mathrm{SNR}}_{\text {gain }}$ as the 'Technology-curve' and $K_{\text {system }}$ as the 'Technology-curve coefficient'.

\section{Requirements and assumptions}

The model requires the link budget to be known for a single set of conditions, typically OOK for a particular data rate and BER. Also, it requires the variation in transmitter power with bandwidth and that in receiver sensitivity with bandwidth (using bandwidth to determine the noise and collection area as dependent variables). For the 'Technology-curve' based design method, the assumptions are that (i) the transmitter bandwidth power variation follows a power law, (ii) the receiver aperture is over-filled by the illumination from the transmitter, so that an increase in receiver area causes a proportionate increase in power, (iii) bandwidth of the receiver is mainly limited by the detector capacitance, and reduction in bandwidth creates an increase in detection area inversely, (iv) receiver noise consists of signal dependent shot-noise and signal independent amplifier noise, as well as signal independent ambient light noise.

These assumptions are reasonable and valid for many practical cases. 'Technology-curve' enables a holistic design method, rather than referring to particular devices. If we consider various device and system parameters, the shape and/or the slope of the 'Technology-curve' may change. Therefore, it may not be able to express the device constraint with purely the single system parameter ' $K_{\text {system }}$ '. However, if it is possible to describe the change in available SNR with bandwidth mathematically, or even graphically, 'Technologycurve' based optimisation still works.

\section{OPTIMISATION USING THE TECHNOLOGY-CURVE}

\section{A. Holistic design}

Fig. 2 shows the available SNR ( $\left.\overline{\mathrm{SNR}}_{\text {gain }}\right)$ from the technology-curves and the required SNR ( $\left.\mathrm{SNR}_{\mathrm{mod}}\right)$ according to the relative $\mathrm{BW}$ for selected modulation schemes. $\mathrm{SNR}_{\text {mod }}$ and relative BW are normalised with respect to those of OOK as follows: $L$-level pulse amplitude modulation $(L$-PAM) [8] and $L$-level pulse position modulation ( $L$-PPM) [8], Asymmetrically clipped optical orthogonal frequency division multiplexing (ACO-OFDM) [15], and a symbol-spaced zero-forcing decision-feedback equaliser in bandlimited Gaussian channel 


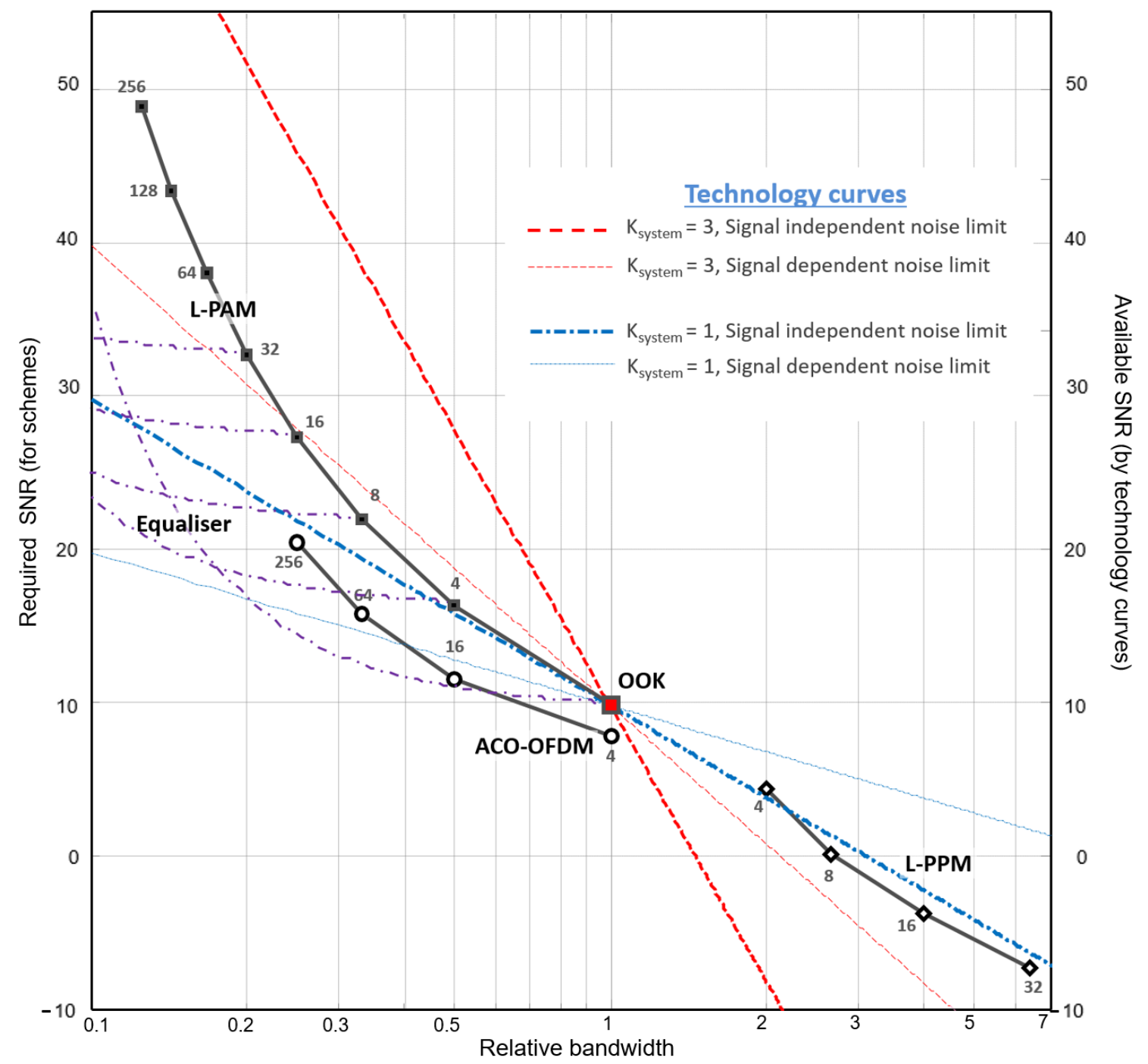

Fig. 2. Relative SNR and BW from Technology-curves and various communication schemes for a BER of $10^{-3}$, for the same data-rate

(a) Optimum L-PAM

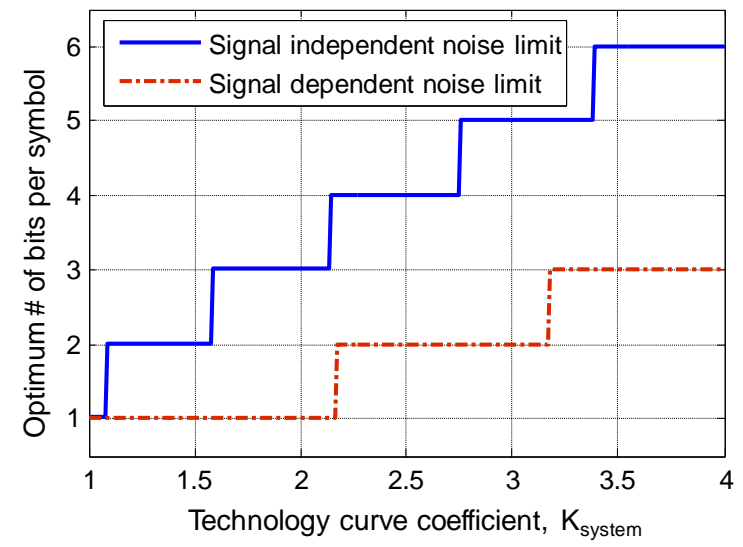

(b) Maximum achievable SNR margin

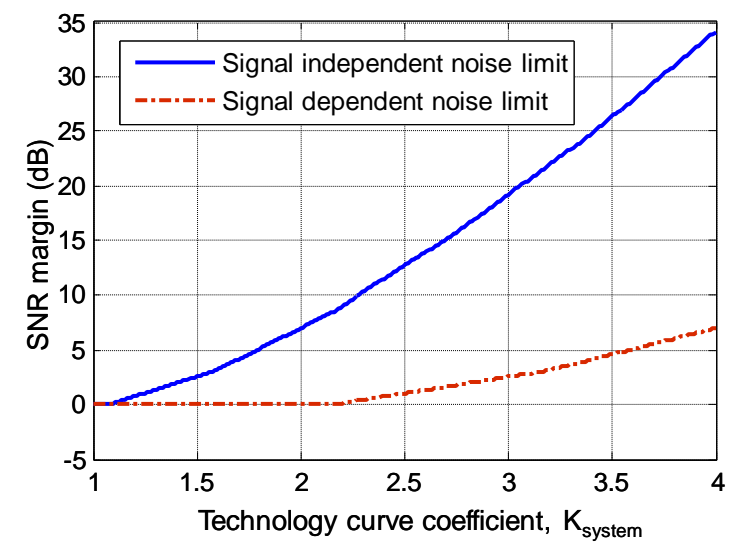

Fig. 3. (a) Optimum number of bits for L-PAM, and (b) achievable SNR margins from Technology-curve with different $K_{\text {system }}$

(Equaliser) [16]. Detailed implementation methods and properties of the schemes can be found in the references. Fig. 2 also shows the technology-curves for different values of $\mathrm{K}_{\text {system. }}$ These are also normalised to $\mathrm{OOK}$, setting $\mathrm{SNR}_{0}$ and $\mathrm{BW}_{0}$ to the values required for OOK (assuming a bit-rate equal to $\mathrm{BW}_{0}$ and an SNR of $9.8 \mathrm{~dB}$ for a BER of $10^{-3}$ ). It can be seen that, depending on $K_{\text {system }}$ and the dominant noise process (signal dependent or independent) there is a considerable increase in available SNR as the $\mathrm{BW}$ reduces. This is due to the characteristics of the transmitter and receiver devices as characterised by $K_{\text {system. }}$. However, the decrease in BW requires more complex multilevel modulation schemes to keep the data 
rate constant, and the required SNR increases as shown in the required SNR points for $L$-PAM and ACO-OFDM scheme. The purple lines indicates the required SNR when the equaliser is applied to the $L$-PAM scheme. Knowing the margin between the available and required SNR allows an optimum modulation scheme to be chosen.

Fig. 3 (a) shows the optimum number of bits per symbol for L-PAM. The optimum level maximising the SNR margin is chosen, given various technology-curve coefficients. Fig. 3 (b) shows the maximum achievable margins for different $K_{\text {system }}$ at these optimum, given $0 \mathrm{~dB}$ margin for 2-PAM. Using the $K_{\text {sys- }}$ tem $=3$ curve for the signal independent noise case the largest gap between the $L$-PAM curve showing the required SNR and the dotted red line showing $K_{\text {system }}$ occurs for 32-PAM, indicating that for a system which had $0 \mathrm{~dB}$ margin for OOK, the margin had increased to close to $\sim 20 \mathrm{~dB}$ for 32 -PAM operating at 5 bits/symbol and a bandwidth of $1 / 5$ of that required for OOK. (This margin is obtained by the extra emission power, increased collection area and lower noise due to reduced receiver bandwidth.) It can be used to either improve the geometric characteristics of the link (such as coverage area), the robustness, or increase the rate. This approach assumes a variable device set, and if bandwidth is fixed the available SNR is known and analyses of the type in [8] are appropriate to determine the maximum rate.

\section{B. (Example-I) Optimisation using the holistic design: Information broadcasting system using a white LED (WLED) illumination.}

In this example, multiple sources broadcast the same information and a single detector is used for signal reception. The aim is to find the optimum device-communication scheme pair and predicting the maximum achievable data-rate. The total luminous flux, the layout of WLEDs and the divergence angle are designed to generate an illumination level $>400$ lux in a typical room [17]. As this is a requirement, the total source power is fixed and $k_{s}=0$.

Table 1. Information broadcasting system design

\begin{tabular}{|c|c|}
\hline \multicolumn{2}{|c|}{$\begin{array}{c}\text { "Given conditions below, design the fastest optical wire- } \\
\text { less broadcasting system in a typical room" }\end{array}$} \\
\hline Source type & WLEDs (phosphor + GaN LED) \\
\hline Illuminance & 400 lux \\
\hline $\begin{array}{c}\text { Detector } \\
\text { Type }\end{array}$ & APD: Hamamatsu, S8664 series, $K_{d}=1.69$ \\
\hline $\begin{array}{c}\text { Additional } \\
\text { constraints }\end{array}$ & $\begin{array}{c}\text { Blue optical filtering loss: } 3 \mathrm{~dB} \\
\text { Signal independent noise: } 1.5 \mathrm{nA} / \sqrt{\mathrm{Hz}}[18]\end{array}$ \\
\hline
\end{tabular}

<Step-1> Establish the reference Technology-curve.

Receivers with area-bandwidth relationships corresponding to Hamamatsu S8664 series detectors are considered, as shown in Fig. 1 (b). Under the conditions stated in Table 1, using the SNR equation in (4.2), it is found that the signal independent noise is dominant. Therefore, the BW and SNR relationship can be characterised by the Technology-curve with $K_{\text {system }}=1.69$ as below.

$$
\overline{S N R}_{\text {gain }}=\Delta \mathrm{BW}_{\text {system }}^{-2 K_{\text {system }}}=\Delta \mathrm{BW}_{\text {system }}^{-3.38}
$$

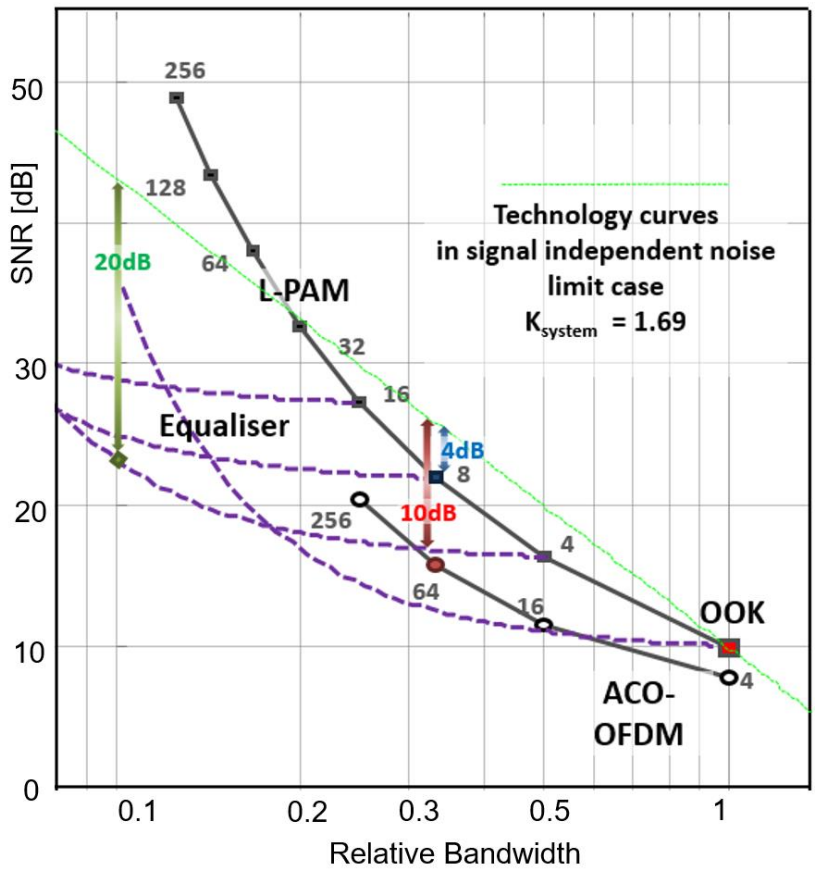

Fig. 4. Relative SNR and BW to find the optimum communication scheme and device pair using Technology-curve

<Step-2> Determine the scheme with the best margin.

Fig. 4 shows the technology-curve and the reference OOK scheme. The reference SNR is $9.8 \mathrm{~dB}$ (for a target bit-error-rate of $10^{-3}$ and bit-rate=BW). It can be seen from Fig. 4 that 8-PAM offers a $4 \mathrm{~dB}$ increase in margin, ACO-OFDM $10 \mathrm{~dB}$, and equalised 4-PAM $20 \mathrm{~dB}$.

<Step-3> Identify the optimum device and scheme pair and the maximum data-rate.

So far, the results are independent of any link geometry, bandwidth, and are purely a function of the technology used for transmitter and receiver-the only link specific assumption made is that there is sufficient SNR $(9.8 \mathrm{~dB})$ to support an OOK link, and that bit-rate=BW. The final stage is to apply the general result and obtain real device parameters. The equalised 4-PAM uses $1 / 10$ the BW of OOK, and the $20 \mathrm{~dB}$ margin found in Step2 can be used to increase the reference OOK data-rate. This is achieved using the conditions in table 1 and applying equation (4.2) to find the actual OOK SNR and finding the data rate at which this is $-10.2(=9.8-20) \mathrm{dB}, 412 \mathrm{Mbit} / \mathrm{s}$ in this example. This means although $412 \mathrm{Mb} / \mathrm{s}$ OOK using $412 \mathrm{MHz} \mathrm{BW}$ cannot be supported as it is, reducing the device $\mathrm{BW}$ to $41.2 \mathrm{MHz}$ creating a $20 \mathrm{~dB}$ SNR gain recovers the SNR. Hence, the 412 $\mathrm{Mb} / \mathrm{s}$ equalised 4-PAM using 41.2 $\mathrm{MHz} \mathrm{BW}$ works with $0 \mathrm{~dB}$ of margin. This data-rate is almost 3 times that of the reference OOK case where the $9.8 \mathrm{~dB} \mathrm{SNR}$ (for $\mathrm{BER}=10^{-3}$ ) is achieved at $140 \mathrm{Mb} / \mathrm{s}$. The optimum detector size $\left(27 \mathrm{~mm}^{2}\right)$ is also found for the BW of $41.2 \mathrm{MHz}$ from Fig. 1 (b). As the channel bandwidth is known, the final step in the design is to fix the number of sources required using power-bandwidth data of the type shown in Fig 1(a).

\section{C. (Example-II) Finding the optimal device from limited choices}

In reality, commercially available receivers have a limited selection of size, gain and noise $\left(A_{d}, G_{r}\right.$ and $\left.N_{\text {ind }}\right)$, so it can be 
difficult to find real devices with precisely optimum values. The concept of Technology-curve based analysis, however, can still be helpful to find the optimal devices by visualising supportable communication schemes and corresponding SNR margins. In this example, a method to choose the optimal receiver from two candidates in a point-to-point link is described. Although it is possible to compare more than two candidates, two were chosen due to the availability of the experimental devices. The target and device properties are summarised in Table 2.

Table 2. Point to point link design

\begin{tabular}{|c|c|c|}
\hline \multicolumn{3}{|c|}{$\begin{array}{l}\text { "For a } 10 \mathrm{Mb} / \mathrm{s} \text { point-to-point link, } \\
\text { find the optimal receiver and L-PAM pair, leading to the min- } \\
\text { imum required irradiance" }\end{array}$} \\
\hline Transmitter & $\begin{array}{l}\text { RC-LED } \\
(\mathrm{RC} 650)\end{array}$ & $\begin{array}{l}\text { Wavelength: } 650 \mathrm{~nm} \\
\text { BW: } 100 \mathrm{MHz} \\
\text { PRC-LED }: 1 \mathrm{~mW} \\
\text { Irradiance by link- } \\
\text { distance (See Fig. 7) }\end{array}$ \\
\hline \multirow{2}{*}{$\begin{array}{l}\text { Receiver } \\
\text { candidates }\end{array}$} & Receiver-I [19] & 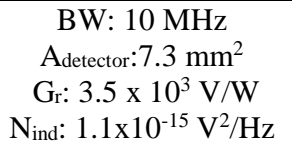 \\
\hline & Receiver-II [20] & $\begin{array}{c}\text { BW: } 3.6 \mathrm{MHz} \\
\text { Adetector: } 150 \mathrm{~mm}^{2} \\
\mathrm{G}_{\mathrm{r}}: 2.5 \times 10^{3} \mathrm{~V} / \mathrm{W} \\
\mathrm{N}_{\text {ind }}: 1.1 \times 10^{-15} \mathrm{~V}^{2} / \mathrm{Hz}\end{array}$ \\
\hline $\begin{array}{l}\text { Additional } \\
\text { requirement }\end{array}$ & $\begin{array}{c}\text { Margin for } \\
+-45 \text { degree FOV }\end{array}$ & $\begin{array}{l}1.5 \mathrm{~dB} \text { (optical) } \\
3 \mathrm{~dB} \text { (electrical) }\end{array}$ \\
\hline
\end{tabular}

For this example, a resonant cavity LED (RC-LED) with radiant power $\left(\mathrm{P}_{\mathrm{RC}-\mathrm{LED}}\right)$ of $1 \mathrm{~mW}$ is considered. There are two candidate receivers with different $\mathrm{BW}$ and the effective area as shown in Table 2. The target is to find the optimal receiver and $L$-PAM pair, leading to the minimum required irradiance for 10 $\mathrm{Mb} / \mathrm{s}$ point-to-point link. The design steps are as follows.

$<$ Step-1> Establish the reference Technology-Curve.

Since the selection of the device is limited, stair-like curves are drawn in Fig. 5. The clear trade-off between BW and SNR of the two candidates shows that receiver-II (indicated with ' $\square$ ') has $23 \mathrm{~dB}$ more SNR than receiver-I (with ' $\diamond$ '), whilst having a $1 / 3$ the $\mathrm{BW}$ of receiver-I.

$<$ Step-2> Determine the scheme and device pair with the highest margin.

The modulation schemes under the curve can be supported by either of the devices, and the difference between the curve and the SNR requirement represents the margin. For instance, receiver-I at point (a) can support only OOK, but receiver-II at (b) can support 8,16 and 32-PAM for the same data-rate. The scheme leading to the highest margin is chosen to be optimal, which is 8-PAM in this example.

$<$ Step-3> Find the minimum required irradiance with the additional requirement of +- 45 degree FOV.

Since the optimum scheme-receiver pair (8-PAM with receiver-II) is known, the minimum required irradiance with the additional margin for the FOV requirement can be calculated.

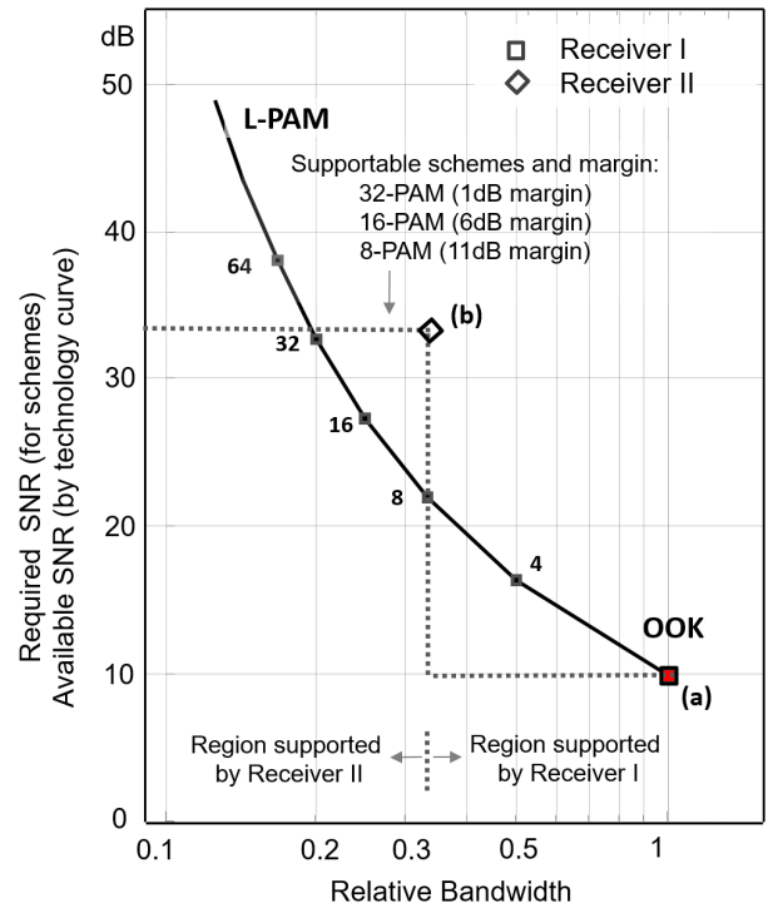

Fig. 5. Relative SNR and BW for L-PAM and Technology-curves to choose the best receiver for the target in Table 2

The irradiance level leading to a $25 \mathrm{~dB}$ SNR ( $22 \mathrm{~dB}$ for 8-PAM and $3 \mathrm{~dB}$ for FOV) is derived to be $0.01 \mathrm{~W} / \mathrm{m}^{2}$. Therefore, although using OOK with receiver-I acquires only $\sim 2 \mathrm{~dB}$ SNR from the same irradiance level, the optimum pair can construct a reliable communication link meeting the target.

To experimentally validate this, first, the irradiance vs distance (Fig. 6 (a)) from an RC-LED with a collimating aspheric lens (Fig. 6 (b)) is measured. It is shown that at $20 \mathrm{~m}$, an irradiance of $0.01 \mathrm{~W} / \mathrm{m}^{2}$ is achieved. Then, using an arbitrary waveform generator (Agilent 81150) modulating the RC-LED with random bit-sequences encoded to 2-PAM (OOK) and 8-PAM.

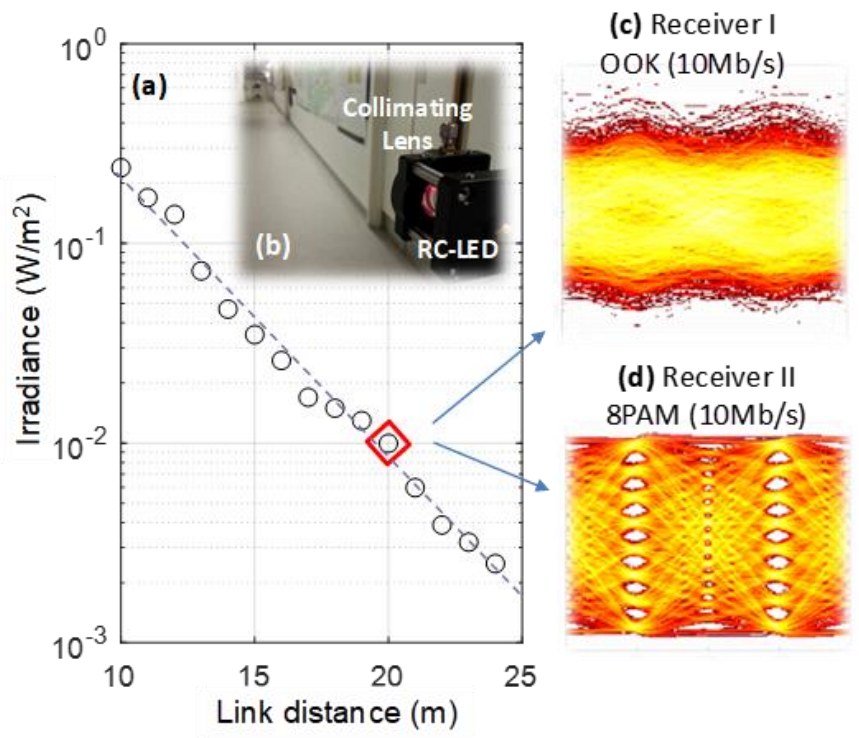

Fig. 6. (a) Measured irradiance $\left(\mathrm{W} / \mathrm{m}^{2}\right)$ by link distance, (b) picture showing the transmitter based on RC-LED, (c) measured $10 \mathrm{Mb} / \mathrm{s}$ OOK eye-diagram using Receiver I and (d) $10 \mathrm{Mb} / \mathrm{s} 8$-PAM eye-diagram using Receiver II. 
After capturing the received signal from an oscilloscope (Agilent MSO6104), the performance of the two receivers at 10 $\mathrm{Mb} / \mathrm{s}$ is tested. As predicted in the optimisation process, whilst the receiver-I with OOK shows a complete eye-closure in the eye-diagram (Fig. 6 (c)) the receiver-II with 8-PAM shows a clear eye-opening (Fig. 6 (d)). This is mainly due to the receiver-II receiving 20 times more signal compared to the receiver-I. There was no error in the bits decoded from the 8-PAM signal. From the received signal statistics, the SNR margin for $10^{-3}$ BER is calculated to be $\sim 3 \mathrm{~dB}$, which is in a good agreement with the predicted value.

\section{CONCLUSIONS}

For the optimum design of an OWC system, a comprehensive modelling approach is essential. This paper achieves this by introducing a 'Technology-curve' which shows the relationship between SNR and BW for a particular device technology set. For the optimum design of an OWC system, a comprehensive modelling approach is essential. This paper achieves this by introducing a 'Technology-curve' which shows the relationship between SNR and BW for a particular device technology set. Previous work such as [8] compare modulation schemes by analysing the bandwidth and power requirements. To complete the optimisation, however, available resources such as SNR and bandwidth provided from devices must be included. To the best of our knowledge, for the first time, a method to incorporate the available resources in the modelling with an effective and intuitive way using a single parameter was introduced. Further, it was shown that the optimum communication scheme is strongly dependent on transmitter and receiver capabilities represented by the 'Technology-curve', and specific examples of optimal link design were presented to validate the approach. Future work includes i) advancing the Technology-curve model by accommodating more practical constraints such as device dynamic range and frequency dependent noise, and ii) adding more communication schemes such as other optical-OFDMs, multi-input-multi-output (MIMO) and wavelength division multiplexing (WDM) schemes.

\section{REFERENCES}

[1] D. C. O'Brien, S. Rajbhandari Sujan and H. Chun, " Transmitter and receiver technologies for optical wireless," Phil. Trans. R. Soc. A. 2020.

[2] H. Haas, C. hen, D. O'Brien, "A guide to wireless networking by light," Progress in Quantum Electronics, Vol.55 , pp 88-111, 2017.

[3] A. Chaaban, et al., "Capacity of optical wireless communication channels," Phil. Trans. R. Soc. 2020.

[4] M. Uysal and H. Nouri, "Optical wireless communications - An emerging technology," 2014 16th International Conference on Transparent Optical Networks (ICTON), Graz, 2014, pp. 1-7.

[5] L. Grobe, et. al., "High-Speed Visible Light Communication Systems," Communications Magazine, IEEE, vol.51, no.12, pp.60,66, December 2013.

[6] S. Rajbhandari, J. J. D. McKendry, J. Herrnsdorf, H., G. Faulkner, H. Haas, I. M. Watson, D. O'Brien, and M. D. Dawson," Gallium Nitride LEDs for multigigabit-per-second visible light data communications,"
IOP, Semiconductor Science Technology, 2016.

J. J. D. McKendry et al., "Visible-Light Communications Using a CMOS-Controlled MicroLight- Emitting-Diode Array," J. Light. Technol., vol. 30, no. 1, pp. 61-67, Jan. 2012.

[8] J. R. Barry, "Wireless Infrared Communications," Springer, 1994, ch. 5.3.

[9] Z. Gong et al., "Size-dependent light output, spectral shift, and self-heating of $400 \mathrm{~nm}$ InGaN light-emitting diodes," J. Appl. Phys., vol. 107, no. 1, p. 013103, 2010.

[10] P. Djahani and J. M. Kahn, "Analysis of infrared wireless links employing multibeam transmitters and imaging diversity receivers," in IEEE Transactions on Communications, vol. 48, no. 12, pp. 2077-2088, Dec. 2000.

[11] G. Yun and M. Kavehrad, "Spot-diffusing and fly-eye receivers for indoor infrared wireless communications," 1992 IEEE International Conference on Selected Topics in Wireless Communications, Vancouver, BC, Canada, 1992, pp. 262-265, doi: 10.1109/ICWC.1992.200761.

[12] D. C. O'Brien et al., "Integrated transceivers for optical wireless communications," in IEEE Journal of Selected Topics in Quantum Electronics, vol. 11, no. 1, pp. 173183, Jan.-Feb. 2005.

[13] Z. Cao et al., "Ultrahigh Throughput Indoor Infrared Wireless Communication System Enabled by a Cascaded Aperture Optical Receiver Fabricated on InP Membrane," IEEE J. Light. Technol., vol. 36, no. 1, pp. 57-67, 2018.

[14] J. Grubor, S. Randel, K.-D. Langer, and J. W. Walewski, "Broadband Information Broadcasting Using LED-Based Interior Lighting," J. Light. Technol., vol. 26, no. 24, pp. 3883-3892, Dec. 2008.

[15] J. Armstrong and B. Schmidt, "Comparison of asymmetrically clipped optical OFDM and DC-biased optical OFDM in AWGN," Commun. Lett. IEEE, vol. 12, no. 5, pp. 343-345, 2008.

[16] S. Randel and F. Breyer, "Advanced modulation schemes for short-range optical communications," Selected Topics in Quantum Electronics, IEEE Journal of , vol.16, no.5, pp.1280,1289, Sept.-Oct. 2010.

[17] H. Chun, S. Rajbhandari, G. Faulkner and D. O'Brien, "Effectiveness of blue-filtering in WLED based indoor Visible light communication," 2014 3rd International Workshop in Optical Wireless Communications (IWOW), Funchal, 2014, pp. 60-64.

[18] S. Rajbhandari, Z. Ghassemlooy and M. Angelova, "Experimental wavelet based denoising for indoor infrared wireless communications," Opt. Express 21, 1377913784, 2013.

[19] K. D. Dambul, D. C. O'Brien and G. Faulkner, "Indoor Optical Wireless MIMO System With an Imaging Receiver," IEEE Photonics Technology Letters, vol. 23, no. 2, pp. 97-99, Jan.15, 2011.

[20] D. O'Brien, "Multi-input multi-output (MIMO) indoor optical wireless communications," 2009 Conference Record of the Forty-Third Asilomar Conference on Signals, Systems and Computers, Pacific Grove, CA, 
2009, pp. 1636-1639. 